\section{Is CPAP effective in reducing blood pressure in minimally symptomatic obstructive sleep apnoea?}

\author{
Jean-Louis Pépin, ${ }^{1,2}$ Jean-François Timsit, ${ }^{3}$ Renaud Tamisier, ${ }^{1,2}$ \\ Patrick Lévy ${ }^{1,2}$
}

Hypertension affects about $25 \%$ of the adult population worldwide. It ranks as the leading chronic risk factor for

\footnotetext{
${ }^{1}$ INSERM U 1042, Laboratoire HP2 Grenoble, Faculté de Médecine, Université Grenoble Alpes, Grenoble, France; ' ${ }^{2}$ Laboratoire EFCR et Sommeil, Pôle Thorax et Vaisseaux, CHU de Grenoble, Grenoble, France; ${ }^{3}$ IAME UMR 1137 INSERM, University Paris Diderot, Paris, France
}

Correspondence to Professor Jean-Louis Pépin, Laboratoire EFCR, CHU de Grenoble, BP217X 38043 Grenoble cedex 09, France; jpepin@chu-grenoble.fr mortality, accounting for $13.5 \%$ of all deaths. Half of all strokes and ischaemic heart disease events are attributable to high blood pressure (BP). ${ }^{12}$

Obstructive sleep apnoea (OSA) is now recognised as a risk factor for the development of hypertension in European and the US International Guidelines. OSA and hypertension are linked in a dose-response fashion. This is true even when the usual confounding factors such as age, alcohol, tobacco consumption and Body Mass Index are taken into account. ${ }^{3}$ In a large prospective observational cohort followed for more than 12 years, it has been shown that compared with controls, the adjusted HRs for incident hypertension were twofold greater among patients with severe OSA who declined continuous positive airway pressure (CPAP) therapy. ${ }^{4}$

At least four meta-analyses derived from 32 randomised controlled trials (RCTs) have demonstrated that CPAP, the first-line therapy for moderate to severe OSA syndrome, reduces $24 \mathrm{~h}$ mean BP by approximately $-2 \mathrm{~mm} \mathrm{Hg}$ (pooled estimated effect). ${ }^{5-8}$ In these meta-analyses, heterogeneity is a clear concern with some of the analysed studies excluding hypertensive patients, while others only included hypertensive patients. Furthermore, the presence and/or the description of antihypertensive classes of medications are poorly reported. The first strength of the study by Bratton et $a l^{9}$ is to restrict the investigation to a homogeneous group with minimally symptomatic OSA. Whereas CPAP treatment is 
clearly indicated in sleepy symptomatic OSA, CPAP prescription with the aim of reducing cardio-metabolic risk in minimally symptomatic patients remains a frequent clinical practice. This should be argued because CPAP effects on hypertension appear to be limited mainly to the more severe and symptomatic patients, with little benefit observed in patients who did not feel sleepy during the day. In their recent meta-analysis, Montesi et $a l^{8}$ reported that with every 5 -point increase in the Epworth Sleepiness Scale score at baseline, a drop in diurnal systolic BP (SBP) of $1.86 \mathrm{~mm} \mathrm{Hg}$ and a drop in diurnal diastolic BP (DBP) of $1.35 \mathrm{mmHg}$ could be expected with CPAP treatment. Thus, the question addressed by the meta-analysis of Bratton et al is clinically relevant and targets a population in which the true efficacy of CPAP on BP is still debated. In summary, they found no beneficial effect on BP in patients with minimally symptomatic OSA, except in those patients who used CPAP for $>4 \mathrm{~h} /$ night.

The meta-analysis uses data obtained for individual patients, which allows analysis of different subgroups, thus identifying those with OSA who are most likely to benefit from CPAP. This is an additional strength, as this methodology allows adjustment for covariates, thus reducing potential bias and increasing power. ${ }^{10} \mathrm{By}$ using the full information in the dataset, this method allows subgroup analyses and adjustment on confounding variables.

However, several aspects of the data analysed and the conclusions should be discussed. First, only daytime or office BP readings were used to assess the impact of CPAP. OSA-related hypertension has several characteristics: diastolic and nocturnal predominance are commonly encountered often leading to masked hypertension and non-dipper status. ${ }^{11}$ Diastolic hypertension is more frequent in OSA as the main mechanism for BP elevation is an increase in vascular resistance owing to sympathetic activation. Due to the nocturnal predominance of hypertension, patients with OSA are at high risk of presenting masked hypertension, that is, normal office BP but elevated BP using $24 \mathrm{~h}$ ambulatory BP monitoring (ABPM). Moreover, it has been recently published that neither office BP measurements nor self-home BP measurements (three morning and three evening BP measurements by the patient at home) were sufficient to detect masked hypertension in patients with OSA, confirming $24 \mathrm{~h} \mathrm{ABPM}$ as the gold standard to detect abnormal BP in patients with OSA. ${ }^{12}$ There is increasing evidence that the mean nocturnal BP level is a major predictor of cardiovascular morbidity and mortality irrespective of $24 \mathrm{~h}$ BP levels. ${ }^{13}$ Thus, the data used in the current meta-analysis, limited to daytime and office BP measurements, does not fully describe the $24 \mathrm{~h}$ effect of CPAP in minimally symptomatic patients with OSA. Bratton et al did not find an overall beneficial effect of CPAP for this OSA subgroup, but the lack of $24 \mathrm{~h}$ measurements may have underestimated significant changes in absolute nocturnal BP values and variability that are key prognostic factors.

Previous meta-analyses agreed on the fact that BP reduction after CPAP initiation is of greater amplitude in patients with severe OSA and in patients who comply with CPAP treatment. Recent interventional RCTs are concordant in suggesting that CPAP adherence $>5.5 \mathrm{~h} /$ night is required to obtain the greatest reduction in $\mathrm{BP} .{ }^{14}$ Accordingly, although there was no overall improvement in BP, Bratton et $a l^{9}$ found that optimal CPAP usage can result in a decrease in DBP. However, the meta-analysis design does not allow to demonstrate a causal relationship between CPAP usage and the size effect of BP reduction. The comparisons between patients with OSA using CPAP treatment $<4$ or $>4 \mathrm{~h} /$ night with controls were not randomised and could, therefore, be due to various confounders that are not controlled. More importantly, CPAP adherence was, by definition, measured after study enrolment and could be a consequence of treatment in itself and not a predictor of good outcome. The definite proof of impact of CPAP therapy on BP in patients for whom compliance is $>4 \mathrm{~h} /$ night needs further RCTs. A screening preinclusion period or the use of a modified intention to treat population based on patients with a good compliance within the first weeks of enrolment might be possible options. The explanatory factors for poor compliance much more than the poor compliance in itself may explain the results. There is a need to conduct future research using individual data from the two largest trials in this meta-analysis to build and test predictive models to determine which patients are most likely to use treatment more, and thus are likely to receive greater benefit in terms of BP reduction.

Shorter sleep duration, sleep fragmentation and alterations in sleep quality are associated with prevalent or incident hypertension even though age, gender, environmental exposure and ethnic disparities are clear confounders. Sleep parameters were not included as covariates in the Bratton et $a l^{9}$ meta-analysis. We know that the selective deprivation in slow wave sleep that is a hallmark of OSA leads to a reduction in the amplitude of nocturnal dipping of $\mathrm{BP}^{15}$ sustaining the hypothesis that disturbed non-rapid eye movement sleep quantity or quality by microarousals might be a mechanism by which an increase of sympathetic tone and, in turn, hypertension appears. Meta-regression analyses of previous meta-analyses ${ }^{8}$ have indeed demonstrated that a greater reduction in BP occurred with higher frequency of arousals during the diagnosis of OSA.

The studies included in the metaanalysis of Bratton $e t a l^{9}$ differed in terms of the type of control treatment (ie, sham-CPAP vs usual care alone) and there was some variation between the individual studies, including the length of follow-up. However, the studies that use subtherapeutic CPAP are relatively small in size and unlikely to alter the main results that mainly come from the larger studies using standard care as a control group.

Generally, the impact of CPAP in reducing $\mathrm{BP}$ is of relatively modest amplitude even in patients with OSA and daytime sleepiness. The merit and the clinical relevance of the Bratton et al ${ }^{9}$ meta-analysis is to demonstrate that the specific subgroup of patients with minimally symptomatic OSA should not be treated with CPAP in an attempt to reduce daytime BP. However, among patients treated for hypertension, even a $1-2 \mathrm{mmHg}$ decrease in BP is already associated with reduced odds of stroke and major cardiovascular events. ${ }^{16}$ Thus, in clinical practice, the advice of the authors is to prescribe a short trial of CPAP treatment to identify those patients who really do use CPAP for more than $4 \mathrm{~h} / \mathrm{night}$, and thus may benefit in terms of a BP reduction. This is probably particularly true in some specific populations that present high cardiovascular risk, such as patients with resistant hypertension. These patients exhibit greater $\mathrm{BP}$ reductions in response to CPAP therapy, ${ }^{17} 18$ and one might expect in these subgroups a greater benefit in longterm outcomes even for minimally symptomatic patients.

Nevertheless, ultimately, CPAP efficacy should be placed in a realistic perspective in comparison with the tangible effects of weight loss or exercise in reducing BP and cardio metabolic risk. ${ }^{19}$ Chirinos et $a l^{20}$ recently randomly assigned 181 patients with OSA to receive treatment with CPAP, a weight-loss intervention, or CPAP plus a 
weight-loss intervention for 24 weeks. In the perprotocol population, the reductions in SBP, mean BP and pulse pressure at 24 weeks were greater in the combined intervention group than in the weight-loss group and the CPAP group. The difference was massive as the reduction in SBP in the combined intervention group was $14.1 \mathrm{~mm} \mathrm{Hg}$ compared with $3.0 \mathrm{~mm} \mathrm{Hg}$ in the CPAP group. ${ }^{20}$ Whereas the majority of respiratory physicians limit their intervention to prescribing CPAP, this study and the Bratton et al ${ }^{9}$ data emphasise the need to offer multiple modalities of treatment to patients with OSA if their cardiovascular and metabolic risk profile is to be successfully modified. After, or in combination with CPAP trial rehabilitation programmes, weight loss reduction programmes should probably be implemented in this population of minimally symptomatic patients with OSA.

Contributors J-LP is responsible for the overall content as guarantor and is involved in the writing and reporting of the work described in this article. J-FT, RT and $P L$ are involved in the writing and reporting of the work described in this article. J-LP and J-FT contributed equally to this manuscript.

Competing interests None.

Patient consent Obtained.

Provenance and peer review Commissioned; internally peer reviewed.

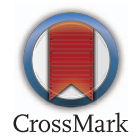

To cite Pépin J-L, Timsit J-F, Tamisier R, et al. Thorax 2014;69:1068-1070.

Published Online First 1 August 2014

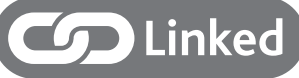

- http://dx.doi.org/10.1136/thoraxjnl-2013-204993

Thorax 2014;69:1068-1070.

doi:10.1136/thoraxjnl-2014-205430

\section{REFERENCES}

1 Kearney PM, Whelton M, Reynolds K, et al. Global burden of hypertension: analysis of worldwide data. Lancet 2005;365:217-23.

2 Lawes CM, Vander Hoorn S, Rodgers A. Global burden of blood-pressure-related disease, 2001. Lancet 2008;371:1513-18.

3 Peppard PE, Young T, Palta M, et al. Prospective study of the association between sleep-disordered breathing and hypertension. N Engl I Med 2000;342:1378-84.

4 Marin JM, Carrizo SJ, Vicente E, et al. Long-term cardiovascular outcomes in men with obstructive sleep apnoea-hypopnoea with or without treatment with continuous positive airway pressure: an observational study. Lancet 2005;365:1046-53.

5 Haentjens P, Van Meerhaeghe A, Moscariello A, et al. The impact of continuous positive airway pressure on blood pressure in patients with obstructive sleep apnea syndrome: evidence from a meta-analysis of placebo-controlled randomized trials. Arch Intern Med 2007;167:757-64.

6 Bazzano LA, Khan Z, Reynolds K, et al. Effect of nocturnal nasal continuous positive airway pressure on blood pressure in obstructive sleep apnea. Hypertension 2007;50:417-23.

7 Alajmi M, Mulgrew AT, Fox J, et al. Impact of continuous positive airway pressure therapy on blood pressure in patients with obstructive sleep apnea hypopnea: a meta-analysis of randomized controlled trials. Lung 2007;185:67-72.

8 Montesi SB, Edwards BA, Malhotra A, et al. The effect of continuous positive airway pressure treatment on blood pressure: a systematic review and meta-analysis of randomized controlled trials. J Clin Sleep Med 2012;8:587-96.

9 Bratton DJ, Stradling JR, Barbé F, et al. Effect of CPAP on blood pressure in patients with minimally-symptomatic obstructive sleep apnoea: a meta-analysis using individual patient data from four randomised controlled trials. Thorax 2014;69: 1128-35.

10 Sauerbrei W, Royston P. A new strategy for meta-analysis of continuous covariates in observational studies. Stat Med 2011;30: 3341-60.

11 Baguet JP, Hammer L, Levy $P$, et al. Night-time and diastolic hypertension are common and underestimated conditions in newly diagnosed apnoeic patients. J Hypertens 2005;23:521-7.

12 Baguet JP, Boutin I, Barone-Rochette G, et al. Hypertension diagnosis in obstructive sleep apnea: Self or 24-hour ambulatory blood pressure monitoring? Int J Cardiol 2013;167:2346-7.

13 Yano Y, Kario K. Nocturnal blood pressure and cardiovascular disease: a review of recent advances. Hypertens Res 2012;35:695-701.

14 Pepin JL, Tamisier R, Barone-Rochette G, et al. Comparison of continuous positive airway pressure and valsartan in hypertensive patients with sleep apnea. Am J Respir Crit Care Med 2010;182: 954-60.

15 Sayk F, Teckentrup C, Becker C, et al. Effects of selective slow-wave sleep deprivation on nocturnal blood pressure dipping and daytime blood pressure regulation. Am J Physiol Regul Integr Comp Physiol 2010;298:R191-197.

16 Turnbull F. Effects of different blood-pressurelowering regimens on major cardiovascular events: results of prospectively-designed overviews of randomised trials. Lancet 2003;362:1527-35.

17 Pedrosa RP, Drager LF, Gonzaga CC, et al. Obstructive sleep apnea: the most common secondary cause of hypertension associated with resistant hypertension. Hypertension 2011;58:811-17.

18 Martinez-Garcia MA, Capote F, Campos-Rodriguez F, et al. Effect of CPAP on blood pressure in patients with obstructive sleep apnea and resistant hypertension: the HIPARCO randomized clinical trial. JAMA 2013;310:2407-15.

19 Pepin JL, Tamisier R, Levy P. Obstructive sleep apnoea and metabolic syndrome: put CPAP efficacy in a more realistic perspective. Thorax 2012;67:1025-7.

20 Chirinos JA, Gurubhagavatula I, Teff K, et al. CPAP, weight loss, or both for obstructive sleep apnea. N Engl J Med 2014;370:2265-75. 